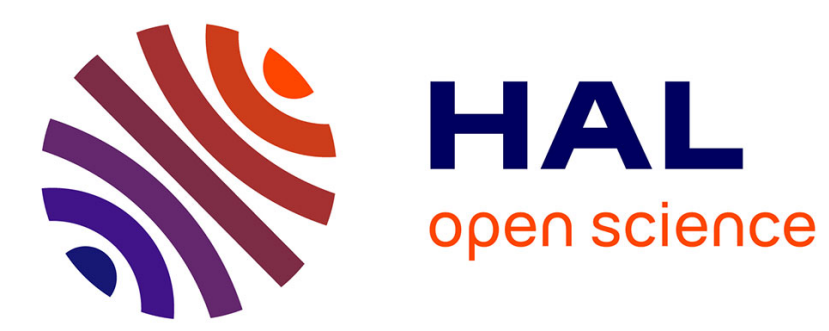

\title{
The large crater on the small asteroid (2867) Steins
}

\author{
M.J. Burchell, J. Leliwa-Kopystynski
}

\section{To cite this version:}

M.J. Burchell, J. Leliwa-Kopystynski. The large crater on the small asteroid (2867) Steins. Icarus, 2010, 10.1016/j.icarus.2010.07.026 . hal-00693824

\section{HAL Id: hal-00693824 \\ https://hal.science/hal-00693824}

Submitted on 3 May 2012

HAL is a multi-disciplinary open access archive for the deposit and dissemination of scientific research documents, whether they are published or not. The documents may come from teaching and research institutions in France or abroad, or from public or private research centers.
L'archive ouverte pluridisciplinaire HAL, est destinée au dépôt et à la diffusion de documents scientifiques de niveau recherche, publiés ou non, émanant des établissements d'enseignement et de recherche français ou étrangers, des laboratoires publics ou privés. 


\section{Accepted Manuscript}

The large crater on the small asteroid (2867) Steins

M.J. Burchell, J. Leliwa-Kopystynski

PII:

S0019-1035(10)00305-2

DOI:

10.1016/j.icarus.2010.07.026

Reference:

YICAR 9523

To appear in:

Icarus

Received Date:

13 January 2010

Revised Date:

26 July 2010

Accepted Date:

28 July 2010

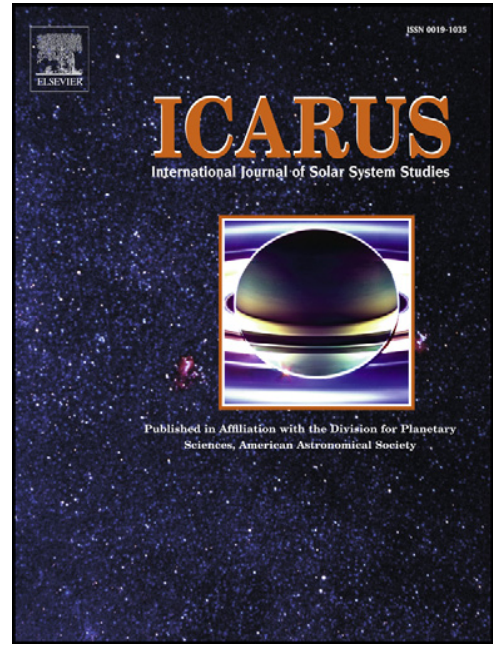

Accepted Date:

Please cite this article as: Burchell, M.J., Leliwa-Kopystynski, J., The large crater on the small asteroid (2867) Steins, Icarus (2010), doi: 10.1016/j.icarus.2010.07.026

This is a PDF file of an unedited manuscript that has been accepted for publication. As a service to our customers we are providing this early version of the manuscript. The manuscript will undergo copyediting, typesetting, and review of the resulting proof before it is published in its final form. Please note that during the production process errors may be discovered which could affect the content, and all legal disclaimers that apply to the journal pertain. 
The large crater on the small asteroid (2867) Steins

M. J. Burchell ${ }^{1}$ and J. Leliwa-Kopystynski ${ }^{2}$

${ }^{1}$ Centre for Astrophysics and Planetary Science, School of Physical Sciences, Univ. of

Kent, Canterbury, Kent CT2 7NH, United Kingdom (email:

M.J.Burchell@kent.ac.uk).

${ }^{2}$ University of Warsaw, Institute of Geophysics, Pasteura 7, 02-093 Warszawa, Poland and Space Research Centre of PAS, Bartycka 18A, 00-716 Warszawa, Poland. (email jkopyst@mimuw.edu.pl).

Number of manuscript pages: 29

Number of figures: 3

Number of tables: 3 
Running header: Large craters on small porous bodies

Corresponding author:

Professor Mark J. Burchell

Centre for Astrophysics and Planetary Science,

School of Physical Sciences,

Univ. of Kent,

Canterbury,

Kent CT2 7NH

United Kingdom.

Email: M.J.Burchell@kent.ac.uk

Phone $+44(0) 1227823248$ 


\section{Abstract}

The maximum size of impact craters on finite bodies marks the largest impact that can occur short of impact induced disruption of the body. Recently attention has started to focus on large craters on small bodies such as asteroids and rocky and icy satellites. Here the large crater on the recently imaged asteroid (2867) Steins (with crater diameter to mean asteroid radius ratio of 0.79 ) is shown to follow a limit set by other similar sized bodies with moderate macroporosity (i.e. fractured asteroids). Thus whilst large, the crater size is not novel, nor does it requires Steins to possess an extremely large porosity. In one of the components of the binary asteroid (90) Antiope there is the recently reported presence of an extremely large depression, possibly a crater, with depression diameter to mean asteroid radius ratio of $\sim(1.4-1.62)$. This is consistent with the maximum size of a crater expected from previous observations of very porous rocky bodies (i.e. rubble-pile asteroids). Finally, a relationship between crater diameter (normalised to body radius) is proposed as a function of body porosity which suggests that the doubling of porosity between fractured asteroids and rubblepile asteroids, nearly doubles the size ( $D / R$ value) of the largest crater sustainable on a rocky body.

Key words: Asteroids, cratering, impact processes. 


\section{Introduction}

The fate of small Solar System bodies under impact is either cratering or disruption. At a critical transition between the two, the largest possible crater will form on the body. The size of such a crater will be a function of several key properties of the body, including its composition and structure. Previous work has looked at impacts on mid-sized icy satellites (diameter $400-1600 \mathrm{~km}$ ) for example (Moore et al., 2004), and impacts on smaller rocky bodies (with diameters of order $10-500 \mathrm{~km}$, e.g. see Thomas 1999). Recently, the data for large impact craters on small rocky and icy bodies (size range $1-500 \mathrm{~km}$ ) was updated by Leliwa-Kopystynski et al., 2008. At these smaller sizes, a range of bodies have been imaged, i.e., asteroids, comets and satellites. These bodies have a variety of compositions; not just rocky and icy, but also of varying porosity. They are also not necessarily spherical, being below the size where gravity will draw the bodies into spherical forms. Nevertheless, LeliwaKopystynski et al., 2008 showed that in the size (diameter) range $1-500 \mathrm{~km}$ the ratio largest crater diameter $(D) /$ body equivalent mean radius $(R)$ was a near constant value (mean $D / R=0.90 \pm 0.05$ averaged over rocky and icy bodies or $D / R=0.84 \pm 0.04$ for just rocky bodies). There is some scatter about these mean values (and the maximum observed values are somewhat larger with $(D / R)_{\max }=1.2$ and 1.6 for icy and rocky bodies respectively), but the data generally lie along a single trend independent of size.

Data from 21 bodies (rocky and icy) were used in the study of LeliwaKopystynski et al., 2008, but more data are becoming available. In particular, there are two new recent reports of large craters on asteroids. One is that of a crater on asteroid (2867) Steins, observed during the fly-by of the Rosetta spacecraft in 2008 (Keller et al., 2010). The initial reports emphasised the presence of a large deep crater 
on the asteroid (e.g. see Besse et al. 2009, Burchell and Leliwa-Kopystynski 2009, Michel et al. 2009). There is also the recent report of a newly detected large depression on (90) Antiope (a binary asteroid with components of 86 and $91 \mathrm{~km}$ diameter) found by observing its light curves. The structure in the light curves has been interpreted as arising due to a bowl shaped depression on the surface of one component and it has been suggested that this may be a crater (Descamps et al., 2009).

Here we consider the observed crater on Steins and compare the crater size to those from other small bodies. We also consider the size of the depression on Antiope and consider what we can learn if we take it as a crater.

\section{(2867) Steins}

Asteroid (2878) Steins has been observed for many years by telescope. It was a target of opportunity for a close fly-by by the Rosetta spacecraft whose main science goal is a rendezvous with comet 67/P Churyumov-Gerasimenko. The Rosetta spacecraft's closest approach to Steins was on Sept. $5^{\text {th }}, 2008$ (Schulz, 2009, Keller et al., 2010). The timing of the encounter meant that the asteroid surface was well lit, permitting extensive optical imaging of the surface (Fig. 1). Prior to the encounter an extensive set of telescope observations had been made of the asteroid, which had permitted a well defined 3-D model of the body to be made based upon the light curve data (e.g., see Fornasier et al., 2006: 2008, Lamy et al., 2008). The results of this work are summarised in Table 1 along with the results of the observations made from the spacecraft during the encounter (Keller et al., 2010). Given its reflectance spectra (Barucci et al., 2005; 2008), the asteroid was held to possess an enstatite like composition and was identified as an E(II) asteroid. 
The images taken during the encounter (Fig. 1 and see Keller et al., 2010), show that Steins has a classical multi-faceted shape, similar to that often found in cut glass. There is a large "hole" in the "top" most facet, and this is interpreted as an impact crater. The dimensions of Steins and its crater can be obtained from the preliminary shape model presented by Besse et al., 2009, and it has a mean radius of $2.7 \pm 0.3 \mathrm{~km}$. An updated model is given by Keller et al., 2010 with mean radius 2.65 $\mathrm{km}$. Since the shape model is not equi-radial on all 3 axes, the individual radii were given as $2.2 \mathrm{~km}$ along its spin axis and $3.1 \mathrm{~km}$ in the perpendicular direction (Keller et al., 2010). These values are equivalent to diameters of 4.4 and $6.2 \mathrm{~km}$, comparable with those estimated pre-encounter (Table 1). The crater was given as having a diameter of $2 \mathrm{~km}$ by Besse et al., 2009 and $2.1 \mathrm{~km}$ by Keller et al., 2010. Based on our analysis of the Rosetta images, it has a maximum diameter of $43 \%$ of the length of the longest axis.

To many people, this seems like a large crater for a small body. The ratio commonly used to characterise relatively large craters on relatively small bodies is the ratio: crater diameter $(D) /$ body radius $(R)$ i.e. $D / R$. Taking $2.1 \mathrm{~km}$ as the crater diameter and a mean radius for Steins of $2.65 \mathrm{~km}$ (as given by Keller et al., 2010), gives a $D / R$ ratio of 0.79 . Since Steins is somewhat oblate, rather than purely spherical, $D / R=0.68$ if the mean equatorial radius is used, or $D / R=0.95$ if the mean polar radius is taken for $R$ (Keller et al., 2010). Alternatively, $D / R=0.86$ if taken directly from the images as suggested above. The possibility that this ratio is relatively large for a small body has prompted attempts to explain it, such as modelling of potential impacts in terms of possible macro or micro porosity of the body (e.g. Michel et al., 2009; Jutzi et al. 2010). However, without quantifying what 
should be considered as a large crater on a small body, this may be somewhat misleading.

In Leliwa-Kopystynski et al., 2008 as well as finding the mean behaviour of $D / R$, a relationship was established for the maximum (limiting) value of the ratio $D / R$, which was 1.2 for an icy body and 1.6 for a rocky body. For example, Deimos (satellite of Mars) has a radius of $6.2 \mathrm{~km}$, a crater diameter of $10 \mathrm{~km}$ and hence $D / R=$ 1.61. Clearly, Steins is well below the limit found by Leliwa-Kopystynski et al., 2008. Indeed it is more akin to the cases of (433) Eros, (951) Gaspra, (5535) Annefrank and Dactyl, with radii of $8.68,6.2,3$ and $0.7 \mathrm{~km}$ and $D / R=0.576,0.48,0.5$, and 0.857 respectively (Fig. 2). Note that these bodies are similar in size to Steins and cover a similar range of values for $D / R$. That the bodies are of similar size to Steins is important. For small rocky bodies the growth of craters and the interaction of the craters with the bulk bodies themselves on a global scale may well depend on the overall size of the body. Some effects, such as global resurfacing after a large impact, are indeed thought to depend on the size of the body (e.g. see Asphaug, 2008 for a discussion).

We can immediately observe that the crater on Steins is not abnormal in terms of its diameter relative to parent body size. Indeed, although there are only a limited number of small rocky bodies which have been observed in sufficient detail to resolve craters, several of these have similar sized craters or larger. Further, the value of $D / R$ for Steins is well away (by a factor of 2) from the rule for limiting behaviour set by Leliwa-Kopystynski et al., 2008.

\section{(90) Antiope}


(90) Antiope is a binary asteroid, located in the main Asteroid Belt. It is considered a member of the Themis family of asteroids, identified by Zappalà et al., 1995 with $\sim 550$ members (completeness size limit $\sim 20 \mathrm{~km}$ ) and more recently reported by Leliwa-Kopystynski et al., 2009 as having over 9000 members (completeness size $\sim 7$ $\mathrm{km}$ and based on analysis of a data set of 205,770 asteroids, compared to 12,487 in Zappalà et al., 1995). Antiope was identified as a synchronous pair by direct imaging (Merline et al., 2000) and was later subject to an extended programme of ground based observations to accurately determine its light curve (Descamps et al., 2007). Based on this campaign the components were identified as having diameters of 91 and $68 \mathrm{~km}$, and orbit the combined centre of mass with a period of $16.505 \mathrm{hrs}$. However, some fine details of the light curves in that work were not fully explained and another extended observing campaign was carried out by Descamps et al., 2009. In this more recent work, the shapes of the components have been corrected to fully explain the data. The new data more fully constrain the tri-axial sizes of the 2 bodies (Table 2) and reveal a large geological feature, diameter $68 \mathrm{~km}$ on one of them. This was described by Descamps et al., 2009, as an impact crater, although given that it has not been directly imaged this is contentious. Further, given the nature of the observations and analysis method it is not possible to determine which of the pair contains the crater. However, here we consider what we learn if we were to take it as a crater. Expressed as a fraction of the mean radii of the two components, this putative crater has $D / R$ between 1.54 and 1.62 (in Descamps et al., 2009 the putative crater diameter was given with respect to the mean diameter as $\sim 0.7$, which is equivalent to $D / R \sim 1.4$ ).

Clearly, whichever of the values of $D / R(1.4,1.54$ or 1.62$)$ is taken, this would one of the largest known craters on a small rocky body (Fig. 2). In terms of $D / R$ this is most comparable with that for Deimos $(D / R=1.61)$, i.e. right on the maximum limit 
previously observed. It is also larger than the value of $D / R=1.26$ obtained for (253)

Mathilde.

\section{Discussion}

When considering the issue of disruption of asteroids, much attention has previously focused on the critical energy density just needed to disruption a body. The impact energy density $Q$ is defined as the kinetic energy of the impactor normalised to the target body mass $\left(\mathrm{J} \mathrm{kg}^{-1}\right)$. The specific value $\left(Q^{*}\right)$ used for critical (i.e. just disrupting impacts) is the value which results in a target whose largest surviving fragment has $0.5 \times$ the initial target mass. Many discussions of asteroid disruption focus on $Q^{*}$ and its evolution with target size, e.g. see Ryan 2000 for a review. Individual papers on the subject also often follow this approach, giving values of $Q^{*}$ or fragment size distributions etc. under different circumstances (e.g. Ryan and Melosh 1998, Fujiwara et al., 1977). By contrast, the limiting crater size considered here is often neglected.

Analytical modelling has considered the question of impact disruption of rocky bodies (e.g. Ryan and Melosh 1998 Love and Ahrens 1996). Again however, the results are dominate by discussion of $Q^{*}$, fragment size distribution etc. One key point however, is that whereas for strength dominated processes, where $Q^{*}$ decreases as target body size increases, at larger sizes gravity dominates and $Q^{*}$ increases as size increases. Depending on the particular model used, this transition occurs for bodies with radii between 0.1 and $10 \mathrm{~km}$, and this is the size range into which Steins falls. However, here this is not an issue, as $Q^{*}$ refers to the disruption and permanent dispersal of the body, whereas it is the disruption alone which sets the size of the maximum observed crater. And again, much of the modelling ignores the maximum 
crater size itself. However, the role of target porosity is often flagged, and is taken as increasing the energy needed for disruption.

The influence of porosity on crater size and collisional disruption has been considered in laboratory experiments of cratering by for example Love et al., 1993. As might be expected, the more porous the target the deeper the crater and similarly, the higher the porosity, the greater the value of $Q^{*}$. However, the just sub-critical maximum crater size was not reported. The role of porosity in crater growth was also reported experimentally by Housen and Holsapple 2003. They found that as porosity increased, ejecta blankets around craters decreased, with more of the material retained in the crater, and the final crater size being dominated by compression of the porous target at the impact site. They then made predictions about ejecta blankets around large craters on 5 small bodies (Deimos, Phobos, Eros, Ida and Mathilde - all considered here as well). They found that that based on their results and assumptions about the composition of Mathilde, then the final size of the crater on Mathilde was potentially the result of compaction of porous material and not ejection of material as was probably the case on the other bodies. Later, Jutzi et al., 2009, included porosity in their hydrocode modelling and reproduced the experimental data for disruption of bodies, but again focused on fragment sizes and ejecta speeds etc. However, by doing this they in effect validated their method of modelling at laboratory scales.

Turning to largest crater sizes, in the literature, it is commonly taken that a crater on a body which has the ratio $D / R>0.75$ is a "giant crater". This is close to $50 \%$ of the maximum value for this ratio reported on small $(<200 \mathrm{~km}$ radius $)$ rocky bodies by Leliwa-Kopystynski et al., 2008. If the $50 \%$ rule were adopted, to be considered a giant crater requires $D / R>0.8$, and $D / R=1.6$ is the physically maximum possible value. Here, the crater on Steins is just at the lower end of the definition of a 
"giant crater". The feature on Antiope by contrast, is right up against the maximum boundary. If any scaling of these limits with the size of the body is considered negligible (Deimos is not too dis-similar in size with Steins) then either the large craters on bodies such as Steins, Eros, Annefrank etc., are still sub-critical (by a factor of two in diameter) or the nature of the target body is playing a critical role in determining the maximum permitted value of $D / R$.

Next we consider if all (or indeed any) of the craters taken here are indeed the maximum that the particular body can sustain. In essence however, this is unknowable. One can approach the problem, as implicitly done here, by taking collections of bodies and using the largest observed cases as representative of the maximum possible. However, is this plausible? If all the bodies were very young for example, then given the cratering rates relevant to their location in the Solar System, it would seem odd if they all possessed maximal craters. There is no reason however to suppose that all the bodies considered here are particularly young. Whilst age does not imply a large impact will have occurred, it does make it more likely. However, having undergone a large impact, we may not be able to accurately determine the age of a body. This is because the crater counts on the object (normally used to help determine its age) may be biased, as the previous craters may well have been in-filled (either directly or by seismic shaking) by the large impact, thus partially re-setting the crater count clock. In some cases age estimates are possible, e.g. for crater Stickney an age of certainly greater than $10^{8}$ and only possibly $10^{9}$ years was suggested based on the ejecta distribution and Phobos' orbital history around Mars (see Thomas 1998).

It is also important that the crater sizes themselves are established. In the case of Stickney, early researchers (e.g. Fujiwara 1991) took the crater size as $11.3 \mathrm{~km}$, whereas here we take the result of Thomas 1998 (who used a more detailed surface 
topology model) of $9.4 \mathrm{~km}$ with an uncertainty of $0.5 \mathrm{~km}$. Another issue that may bias the data concerns the age of the craters themselves and any relaxation that they may have undergone since formation. This will change the $D / R$ value, potentially altering the results of any statistical analysis, i.e. newly cratered bodies may have a different observed $D / R$ ratios than bodies with older craters. In the case of Stickney for example, there is some evidence that the crater has undergone mass-flow, with partial in-filling (e.g. Shingareva and Kuzmin 2001). Part of this is assigned to subsequent impacts on or near the rim of Stickney. The composition of the body other than porosity may also play a role in determining the size of the largest possible crater. Regarding this, we note that Leliwa-Kopystynski et al., 2008 compared large craters on a sample of 22 rocky and icy bodies, and the limiting crater size differed by only $25 \%$, even though the composition varied from rocky to icy. Inside a data set confined to only rocky bodies (of various sorts), the variation may well be smaller. Another point to consider is that even if impact rates etc. are similar, then smaller bodies will be more likely to have undergone a near critical impact before larger ones on average. However, here the size range of the bodies being considered is relatively small so this can be neglected. As regards the other points, there is nothing that would preclude the possibility that the craters are indeed near critical, although it clearly cannot be demonstrated.

We thus ignore the possibility that the craters are significantly sub-critical or that $D / R$ depends on the nature of the rocky body. Then we can fit the data in Fig 2 (the 10 rocky bodies from Leliwa-Kopystynski et al., 2008 plus the two new bodies here - see Table 3 for a summary of the relevant data) and obtain:

$$
D=-(0.17 \pm 0.10)+(1.01 \pm 0.08) R, \text { with } r=0.974 \text {, }
$$


where $r$ is the regression coefficient of the fit. This is for $0.7 \mathrm{~km}<R<120 \mathrm{~km}$. If we take the constant term as negligible, we thus obtain a mean $D / R \sim 1.0$ at these sizes (note that at smaller $R$ values than here more data is required as the constant term is no longer negligible).

However, even if we assume that the craters are all just critical, it may not be justified to ignore the influence of the properties of the various bodies. For example, based upon its reflectance spectra Steins is considered an E(II) type asteroid (Barucci et al., 2005; 2008, Keller et al., 2010). If enstatite is taken as the dominant composition of the interior, then we note that a density of $\sim 3640 \mathrm{~kg} \mathrm{~m}^{-3}$ is reported for enstatite chondrite meteorites, suggesting a microporosity of a few \% (Macke et al., 2009). However, whilst a shape model exists for the body, its mass is not yet determined, so no estimate of the overall porosity can be obtained. Densities and porosities are known for some of the rocky bodies similar in size to Steins and with giant craters with similar $D / R$ ratios. For Eros, the total porosity is given as 0.29 with macroporosity 0.18 (Britt et al., 2002) and $D / R=0.576$. For 243 Ida, the total porosity is given as $0.25-0.36$ with macroporosity $0.16-0.24$ (Britt et al., 2002) and $D / R=$ 0.879. The total porosities are above those suggested for the microporosity of the relevant materials suggesting some macroscopic porosity, but they are not extreme cases and are considered as fractured asteroids (see Britt et al., 2002). The data for $D / R$ and porosity (Table 3) are plotted in Fig. 3, and these bodies seem to form a group with $D / R \sim 0.6-0.9$. The implication is that Steins would be a member of this group.

By contrast, Antiope is a binary pair which permits an estimate of its mass and hence, given the shape model, density which is reported as $1280 \pm 40 \mathrm{~kg} \mathrm{~m}^{-3}$ (Descamps et al., 2009). It is a C-type, carbonaceous asteroid, as is Mathilde. Given 
the low density for Antiope, which is similar to Mathilde $\left(1300 \pm 200 \mathrm{~kg} \mathrm{~m}^{-3}\right)$ this suggests a similar total porosity, which for Mathilde is $0.44-0.61$ with macroporosity 0.34 - 0.46 (Britt et al., 2002). Descamps et al., 2007 suggest a total porosity for Antiope of $\sim 0.5$ (and by implication based on the example of Mathilde, we infer a macroporosity of $\sim 0.4)$. Interestingly Mathilde also has a giant crater, with $D / R=$ 1.26. The density of Deimos is estimated as $2247 \pm 251 \mathrm{~kg} \mathrm{~m}^{-3}$ with total porosity 0.26 -0.74 and macroporosity $0.15-0.63$ (Britt et al., 2002), and its giant crater has $D / R=$ 1.61. The data for $D / R$ and porosity from Table 3 are shown on Fig. 3 , and it is possible to group Deimos, Antiope and Mathilde together as highly porous bodies (loosely consolidated, rubble-pile asteroids) which contain giant craters (or in Antiope's case, a putative crater) near a maximum limit of $D / R \sim 1.6$.

In Fig. 3, the data for normalised crater size vs. porosity not only seems to fall into two groups, but could be interpreted as following general trends. Accordingly, the data were fit using a weighted linear model. The results (for the 5 bodies Deimos, Eros, Ida, Mathilde and Antiope) of fitting $D / R$ vs. total porosity ( $\left.\Psi_{\text {total }}\right)$ and macroporosity $\left(\Psi_{\text {macro }}\right)$ are:

$$
D / R=-(0.62 \pm 0.10)+(4.0 \pm 0.4) \times \Psi_{\text {total }} \text {, with } r=0.892,
$$

and

$$
D / R=-(0.21 \pm 0.09)+(4.1 \pm 0.4) \times \Psi_{\text {macro }}, \text { with } r=0.943 \text {, }
$$

where $r$ is the regression coefficient of the fits. Note that in the fits we initially excluded Phobos. This is because Phobos is often described, based on its porosity, as a "rubble pile" body (e.g. Britt et al., 2002). However, here based on its value of $D / R$ it would more naturally fall into the "fractured" body data set, indicating a degree of uncertainty about its nature. If we repeat the above fits including Phobos, the fit coefficients are all well within $1 \sigma$ of those given above (except for the intercept in the 
macroporosity case, which changes by just over $1 \sigma$ ). It could thus be that Phobos is a fractured body (based on $D / R$ ) or the observed crater, whilst large, is not the maximum size physically possible (based on its porosity determining that it is a "rubble-pile" like body). When more data become available, it will be interesting to see if the data points in Fig. 3 remain in two distinct groups, or follow the trends above given with a more continuous distribution.

\section{Conclusion}

We have added the example of Steins to the data set for large craters on small rocky bodies. In the case of Steins, the crater is indeed a "giant crater" but not an extreme case of such. By contrast, if the large feature reported on Antiope were indeed a crater, then it is an extreme case. By considering the porosity of the parent bodies, we suggest that the definition of what the maximum size crater can be on a particular small body depends on the porosity of the body. This in turn suggests that Steins is a moderately porous body in line with Eros and Ida and is hence a fractured asteroid, whereas Antiope is a loosely consolidated, rubble-pile asteroid.

These assertions are based on a set of assumptions, for example old craters may have relaxed and now possess a slightly different radius to that when formed, etc. More important however, is the requirement that the crater on Steins is the largest that can be supported on that body; if it were not the porosity could be greater. However, if it is the largest possible crater, then the macroporosity of Steins is of order $20 \%$.

We note that Keller et al., 2010, describe Steins as a rubble-pile body. They consider that the $D / R$ value for Steins is high for a body of its size, but as we observe here that is not the case. And in our analysis, if the observed crater is maximal, then the required porosity may arise from heavy fracturing (evidence for large fractures is 
reported by Keller et al., 2010) rather than the need for a complete rubble-pile. Recently, using their model which they had previously validated against laboratory experiments (see above and Jutzi et al., 2009), Jutzi et al., 2010 modelled impacts on a body the size of Steins, under a variety of assumptions as to its composition. They concluded that to sustain a crater this size, Steins must either be a rubble pile (which also features microporosity), or a monolithic body which may or may not contain microporosity.

By contrast to Steins, we find that given that the porosity of Antiope has already been estimated, then the observed feature on Antiope is almost right on the limit of crater size that can be supported on a body with such a low density.

We also observe what may be a trend in the maximum value of the ratio $D / R$ on small rocky bodies as a function of porosity. Given only a limited number of data points we find that, for the range of porosities considered here, doubling the porosity (total or macro-) of a body, nearly doubles the maximum possible value of $D / R$, increasing it by a factor of $\sim 1.6-1.9$. As more data becomes available it should become clearer if there is a continuous population of bodies on a plot of $D / R$ vs. porosity, or if the bodies do indeed split into two groups (fractured and rubble-pile asteroids) as here. The next Rosetta fly-by of an asteroid is scheduled to be (21) Lutetia in July 2010 (Schulz, 2009). The nature of this asteroid is still somewhat uncertain, although it has many features of a C-type asteroid (Lazzarin et al., 2009), but it is considered to have a long exposure history to impacts, it may well therefore contain another large crater to add to the data set. 
Acknowledgements: MJB holds a grant from STFC (UK) and JLK was supported by a grant N203 007 32/0576 provided by MEiN (Poland). We are grateful to the anonymous referees for their helpful comments. 


\section{References}

Asphaug, E., 2008. Critical crater diameter and asteroid impact seismology. Meteoritics and Planetary Science 43, 1075-1084.

Barucci, M.A., Fulchignoni, M., Fornasier, S., Dotto, E., Vernazza, P., Birlan, M., Binzel, R.P., Carvano, J., Merlin, F., Barbieri, C., Belskaya, I., 2005. Asteroid target selection for the new Rosetta mission baseline: 21 Lutetia and 2867 Steins. Astron. \& Astrophys. 430, 313-317.

Barucci, M.A., Fornasier, S., Dotto, E., Lamy, P., Jorda, L., Groussin, O., Brucato, J.R., Carvano, J., Alvarez-Candal, A., Cruikshank, D., Fulchignoni, M., 2008. Asteroids 2867 Steins and 21 Lutetia: Surface composition from far infrared observations with the Spitzer space telescope. Astron. \& Astrophys. 477, 665670.

Besse, S., Groussin, O., Jorda, L., Lamy, P., Kaasalainen, M., Gesquiere, G., Remy, E., and the OSIRIS Team, 2009. 3-Dimensional Reconstruction Of Asteroid 2867 Steins. 40 ${ }^{\text {th }}$ Lunar and Planetary Science Conf., CD-ROM abstract 1545.

Britt, D.T., Yeomans, D., Housen, K., Consolmagno, G., 2002. Asteroid density, porosity, and structure. In: Bottke, W.F., Cellino, A., Paolicchi, P., Binzel, R. (Eds.), Asteroids III. Univ. Arizona Press, Tucson, pp. 485-500. 
Burchell, M. J., Leliwa-Kopystynski, J., 2009. The large crater on asteroid Steins: Is it abnormally large? $40^{\text {th }}$ Lunar and Planetary Science Conf., CD-Rom abstract 1525.

Descamps, P., Marchis, F., Michalowski, T., Vachier, F., Colas, F., Berthier, J., Assafin, M., Dunckel, P.B., Polinska, M., Pych, W., Hestroffer, D., Miller, K., Vieira-Martins, R., Birlan, M., Teng-Chuen-Yu, J.-P., Peyrot, A., Payet, B., Dorseuil, J., Léonie, Y., Dijoux, T., 2007. Figure of the double Asteroid 90 Antiope from AO and lightcurves observations. Icarus 187, 482-499.

Descamps P., Marchis F., Michalowski T., Berthier J., Pollock J., Wiggins P., Birlan M., Colas F., Vachier F., Fauvaud S., Fauvaud M., Sareyan J.-P., Pilcher F., Klinglesmith D.A., 2009. A Giant Crater on 90 Antiope? Icarus 203, 102-111.

Fornasier, S., Belskaya, I., Fulchignoni, M., Barucci, M.A., Barbieri, C., 2006. First albedo determination of 2867 Steins, target of the Rosetta mission. Astron. Astrophys. 449, L9-L12.

Fornasier, S., Migliorini A., Dotto E., Barucci M.A., 2008. Visible and near infrared spectroscopic investigation of E-type asteroids, including 2867 Steins, a target of the Rosetta mission. Icarus 196, 119-134.

Fujiwara, A., 1991. Stickney forming impact on Phobos - crater shape and induced stress distribution. Icarus 89, $384-391$. 
Fujiwara, A., Kamimoto, G., Tsukamoto, A., 1977. Destruction of basaltic bodies by high-velocity impact. Icarus 31, 277-288.

Housen K.R., Holsapple K.A., 2003. Impact cratering on porous asteroids. Icarus 163, $102-119$.

Jutzi, N., Michel, P., Hiraoka, K., Nakamura, A.M., Benz, W., 2009. Numerical simulations of impacts involving porous bodies II. Comparison with laboratory experiments. Icarus 201, 802-813

Jutzi, N., Michel, P., Benz, W., 2010. A large crater as a probe of the internal structure of the E-type asteroid Steins. Astronomy and Astrophysics 509(L2), DOI: $10.1051 / 0004-6361 / 200913549$.

Keller, H.U., Barbieri, C., Koschny, D. Lamy, P., Rickman, H., Rodrigo, R., Sierks, H., A’Hearn, M.F., Angrilli, F., Barucci, M.A., Bertaux, J.L., Cremonese, G., Da Deppo, V., Davidsson, B., De Cecco, M., Debei, S., Fornasier, S., Fulle, M., Groussin, O., Gutierrez, P.J., Hviid, S.F., Ip, W.-H., Jorda, L., Knollenberg, J., Kramm, J.R., Kührt, E., Küppers, M., Lara, L.-M. Lazzarin, M., Lopez Moreno, J., Marzari, F., Michalik, H., Naletto, G., Sabau, L., Thomas, N., Wenzel, K.-P., Bertini, I., Besse, S., Ferri, F., Kaasalainen, M., Lowry, S., Marchi, S., Mottola, S., Sabolo, W., Schröder, S.E., Spjuth, S., Vernazza, P., 2010. E-Type Asteroid (2867) Steins as Imaged by OSIRIS on Board Rosetta. Science 327(5962), 190193. 
Lamy, P., Jorda, L., Fornasier, S., Groussin, O., Barucci, M.A., Carvano, J., Dotto, E., Fulchignoni,M., Toth, I., 2008. Asteroid Steins. III. Spitzer space telescope observations, size determination and thermal properties. Astron. \& Astrophys. 487, 1179-1185.

Lazzarin, M., Marchi, S., Moroz, L. V., Magrin, S., 2009. New visible spectra and mineralogical assessment of (21) Lutetia, a target of the Rosetta mission. Astronomy and Astrophysics 498, $301-311$.

Leliwa-Kopystynski J., Burchell M.J. and Lowen D., 2008, Impact cratering and break up of the small bodies of the Solar System. Icarus 195, 817-826.

Leliwa-Kopystynski J., Burchell M.J., Włodarczyk I., 2009. The Impact Origin Of Eunomia And Themis Families. Meteoritics and Planetary Science 44(12), 1929-1936.

Love, S.G., Hörz, F., Brownlee, D.E., 1993. Target porosity effects in impact cratering and collisional disruption. Icarus 105, 216 - 224.

Love, S.G., Ahrens T.J., 1996. Catastrophic Impacts on Gravity Dominated Asteroids. Icarus $124,141-155$.

Macke R.J., Britt D.T., Consolmagno G.J., 2009. Enstatite Chondrite Physical Properties: Density, Porosity And Magnetic Susceptibility. $40^{\text {th }}$ Lunar and Planetary Science Conf., CD-ROM abstract 1598. 
Merline, W.J., Close, L.M., Dumas, C., Shelton, J.C., Menard, F., Chapman, C.R., Slater, D.C., 2000. Discovery of companions to Asteroids 762 Pulcova and 90 Antiope by direct imaging. Bull. Am. Astron. Soc. 32, 1017.

Michel, P., Jutzi, M., Benz, W., 2009. Reproducing the Big Crater on the Asteroid Steins by Impact Simulations: Implications on the Asteroid's Internal Structure. American Astronomical Society, DPS meeting \#41, \#59.05.

Moore, J.M., Schenk, P.M., Bruesch, L.S., Asphaug, E., McKinnon, W.B., 2004. Large impact features on middle-sized icy satellites. Icarus 171, 421-443.

Ryan, E.V., 2000. Asteroid fragmentation and evolution of asteroids. Ann. Rev. Earth. Planet Sci. 28, $367-389$.

Ryan, E.V., Melosh, H.J., 1998. Impact fragmentation from the laboratory to asteroids. Icarus $133,1-24$.

Schulz, R., 2009. Rosetta-One Comet Rendezvous and two Asteroid Fly-Bys. Solar System Research 43, $343-352$.

Shingareva, T.V., Kuzmin, R.O., 2001. Mass-Wasting Processes on the Surface of Phobos. Solar System Research 35, 431-443.

Thomas, P.C., 1998. Ejecta emplacement on Martian satellites. Icarus 131, 78-106. 
Thomas, P.C., 1999. Large craters on small objects: Occurrence, morphology and effects. Icarus 142, 89-96.

Zappalà, V., Bendjoya, Ph., Cellino, A., Farinella, P., Froeschlé, C., 1995. Asteroid families: Search of a 12,487-asteroid sample using two different clustering techniques. Icarus 116, 291-314. 
Figures

Fig. 1. Images of Asteroid Steins, taken from the Rosetta spacecraft by the OSIRIS imaging system. Image source: ESA web site (ESA C2008 MPS for OSIRIS Team MPS/UPD/LAM/IAA/RSSD/INTA/UPM/DASP/IDA).

Fig. 2. Diameter of largest crater vs. radius of parent body. Data sources: open triangles (Leliwa-Kopystynski et al., 2008), closed square is (2867) Steins and open circle is (90) Antiope. Two curves are shown. The solid line is a fit to all 12 data points (see main text and Table 3), the dashed line is the maximum limit for $D / R$ for rocky bodies from Leliwa-Kopystynski et al., 2008. The value for Steins can be seen to be compatible with the rest of the data set and below the limit (dashed line), whereas that for Antiope is an extreme case comparable to Deimos.

Fig. 3. Diameter of largest crater/radius of parent body plotted against porosity. (a) Total porosity (b) Macroporosity The data seem to separate into two groups, one relatively low porosity group of Fractured Asteroids which has $D / R \sim 0.6-0.9$ and the other, loosely consolidated rubble-pile asteroids, with greater porosity and which observe an upper limit of $D / R=1.6$. The fit lines on both graphs are described in the text. 
Table 1; Properties of (2878) Steins observed pre-encounter (Lamy et al., 2008) and during encounter (Keller et al., 2010). The associated values of the ratio of the diameter of the largest crater over the mean radius of the body $(D / R)$ are also given, assuming $D=2.1 \mathrm{~km}$ as given by Keller et al., 2010 .

\begin{tabular}{lll}
\hline Property & Pre-encounter & Value \\
& (Lamy et al., 2008) & From the encounter \\
& (Keller et al., 2010) \\
\hline Rotation Period (hrs) & $6.04681 \pm 0.00002$ & $6.04679 \pm 0.00002$ \\
Overall dimensions of & $2 \mathrm{a}=5.73 \pm 0.52$, & $2 \mathrm{a}=6.67$ \\
3D shape model $(\mathrm{km})$ & $2 \mathrm{~b}=4.95 \pm 0.45$, & $2 \mathrm{~b}=5.81$ \\
& $2 \mathrm{c}=4.58 \pm 0.41$ & $2 \mathrm{c}=4.47$ \\
Axis ratios & $a / b=1.16 ; a / c=1.25$ & $a / b=1.15 ; a / c=1.49$ \\
Volume $\left(\mathrm{km}^{3}\right)$ & 64.3 & - \\
Mean radius $(\mathrm{km})$ & $2.46 \pm 0.03$ & 2.65 \\
$D / R$ & 0.85 & 0.79 \\
\hline
\end{tabular}


Table 2; Properties of (90) Antiope (Descamps et al., 2009) and possible range of values of the ratio of the diameter of the largest crater over the radius of the body $(D / R)$.

\begin{tabular}{ll}
\hline Property & Value \\
\hline Orbital period $(\mathrm{hrs})$ & $16.5051 \pm 0.0001$ \\
Tri-axial ellipsoid dimensions $(\mathrm{km})$ & Component $\mathrm{A}: \mathrm{a}=46.5, \mathrm{~b}=43.5, \mathrm{c}=41.8$ \\
& Component $\mathrm{B}: \mathrm{a}=44.7, \mathrm{~b}=41.4, \mathrm{c}=39.8$ \\
Total mass $(\mathrm{kg})$ & $(8.3 \pm 0.2) \times 10^{17}$ \\
Bulk density $\left(\mathrm{kg} \mathrm{m}^{-3}\right)$ & $1280 \pm 40$ \\
$D / R$ & $1.46-1.52$ \\
\hline
\end{tabular}


Table 3. Relevant parameters of the rocky bodies discussed in the text and used in

Figures 2 and 3. Note that we have assumed Thebe (Jovian satellite) is a rocky body although its density is not well defined.

\begin{tabular}{|c|c|c|c|c|c|c|}
\hline $\begin{array}{l}\text { Rocky satellite or } \\
\text { asteroid }\end{array}$ & $\begin{array}{c}\text { Radius } \\
R, \mathbf{k m}\end{array}$ & $\begin{array}{c}\text { Density } \rho, \\
\text { kg m}^{-3}\end{array}$ & $\begin{array}{c}\text { Crater } \\
\text { diameter } \\
D, \mathbf{k m}\end{array}$ & $D / R$ & $\begin{array}{c}\text { Total } \\
\text { porosity } \\
\Psi_{\text {total }}\end{array}$ & $\begin{array}{c}\text { Macro- } \\
\text { porosity } \\
\Psi_{\text {macro }}\end{array}$ \\
\hline Phobos & 11.1 & $1867 \pm 76$ & 9.4 & 0.847 & $0.41-0.46$ & $0.29-0.33$ \\
\hline Deimos & 6.2 & $2247 \pm 251$ & 10 & 1.613 & $0.26-0.74$ & $0.15-0-63$ \\
\hline Thebe & 49.3 & 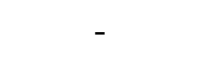 & 44 & 0.892 & - & - \\
\hline 433 Eros & 8.68 & $2670 \pm 30$ & 5 & 1.739 & 0.29 & 0.18 \\
\hline 243 Ida & 15.7 & $2600 \pm 500$ & 13.8 & 0.879 & $0.25-0.36$ & $0.16-0.24$ \\
\hline 253 Mathilde & 26.5 & $1300 \pm 200$ & 33.4 & 1.260 & $0.44-0.61$ & $0.34-0.46$ \\
\hline 951 Gaspra & 6.2 & - & 3 & 0.48 & - & - \\
\hline 3 Juno & $\approx 120$ & 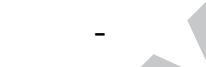 & $>100$ & $\approx 0.83$ & - & - \\
\hline 5535 Annefrank & $\approx 3$ & & $\approx 1.5$ & $\approx 0.50$ & - & - \\
\hline Dactyl (Ida's sat.) & $\approx 0.7$ & & $\approx 0.6$ & $\approx 0.857$ & - & - \\
\hline 2867 Steins & 2.65 & & 2.1 & 0.79 & - & - \\
\hline 90 Antiope (binary) & $44 ; 42$ & $80 \pm 40$ & 68 & $1.4-1.62$ & 0.50 & 0.40 \\
\hline
\end{tabular}




\section{ACCEPTED MANUSCRIPT}

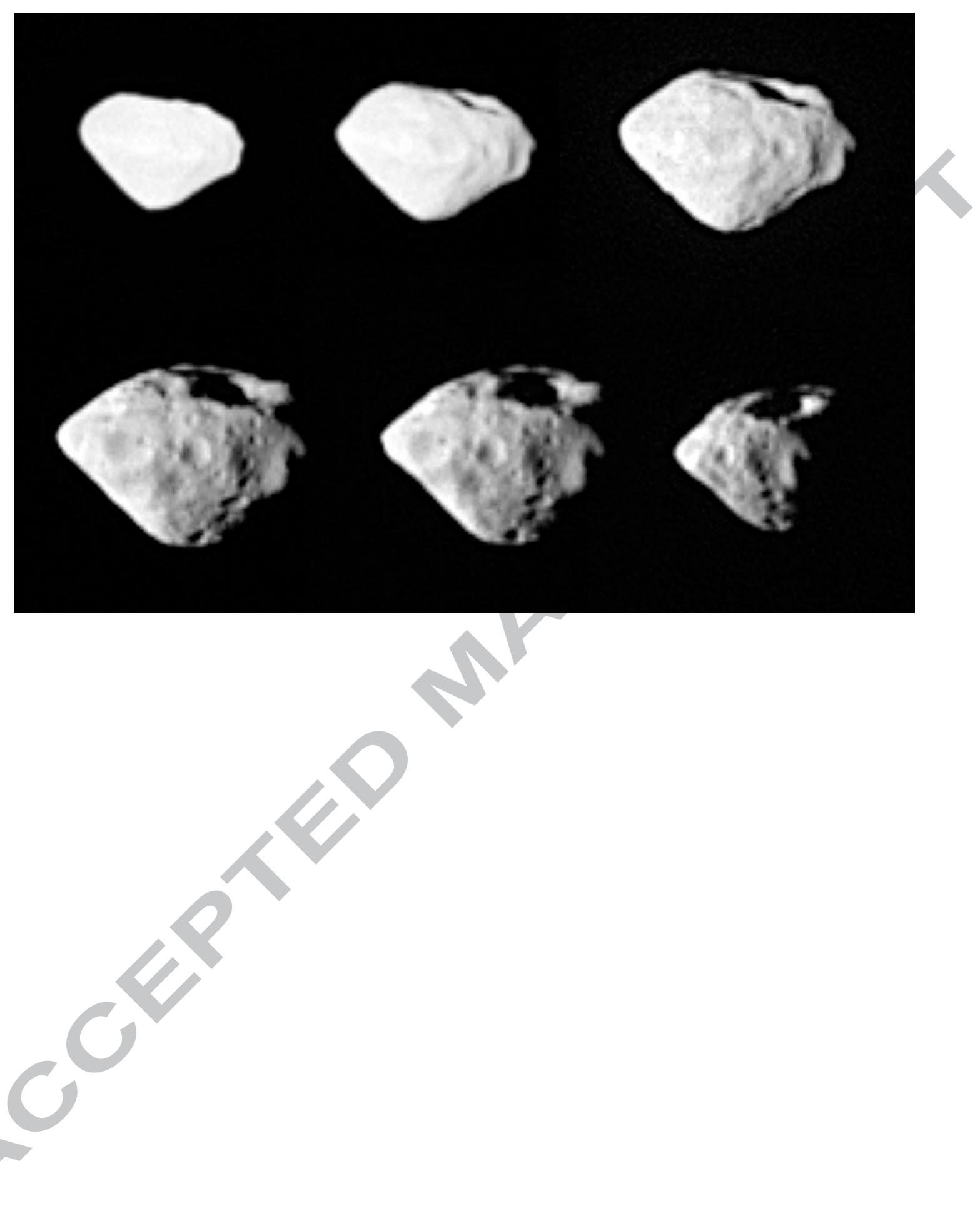




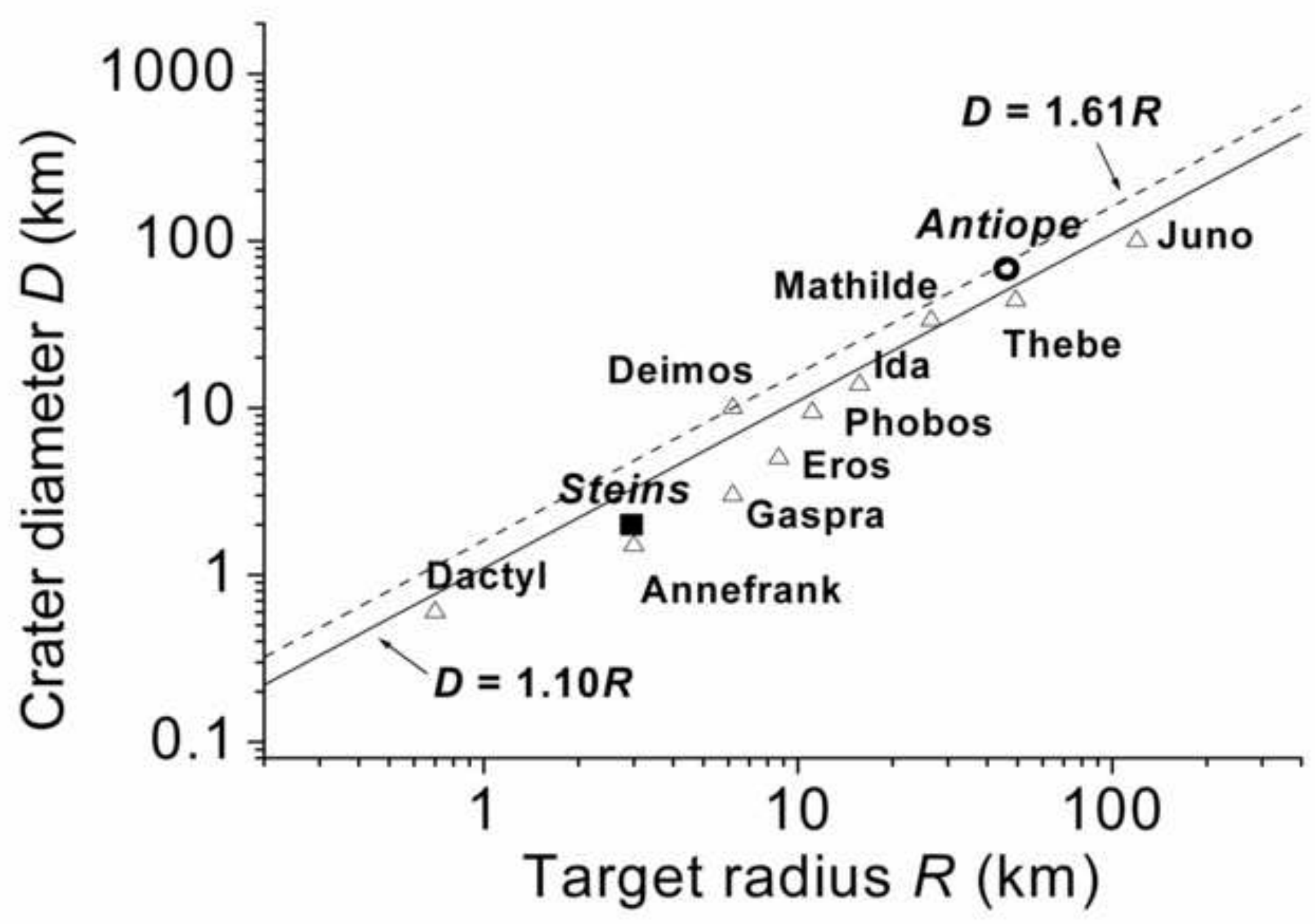




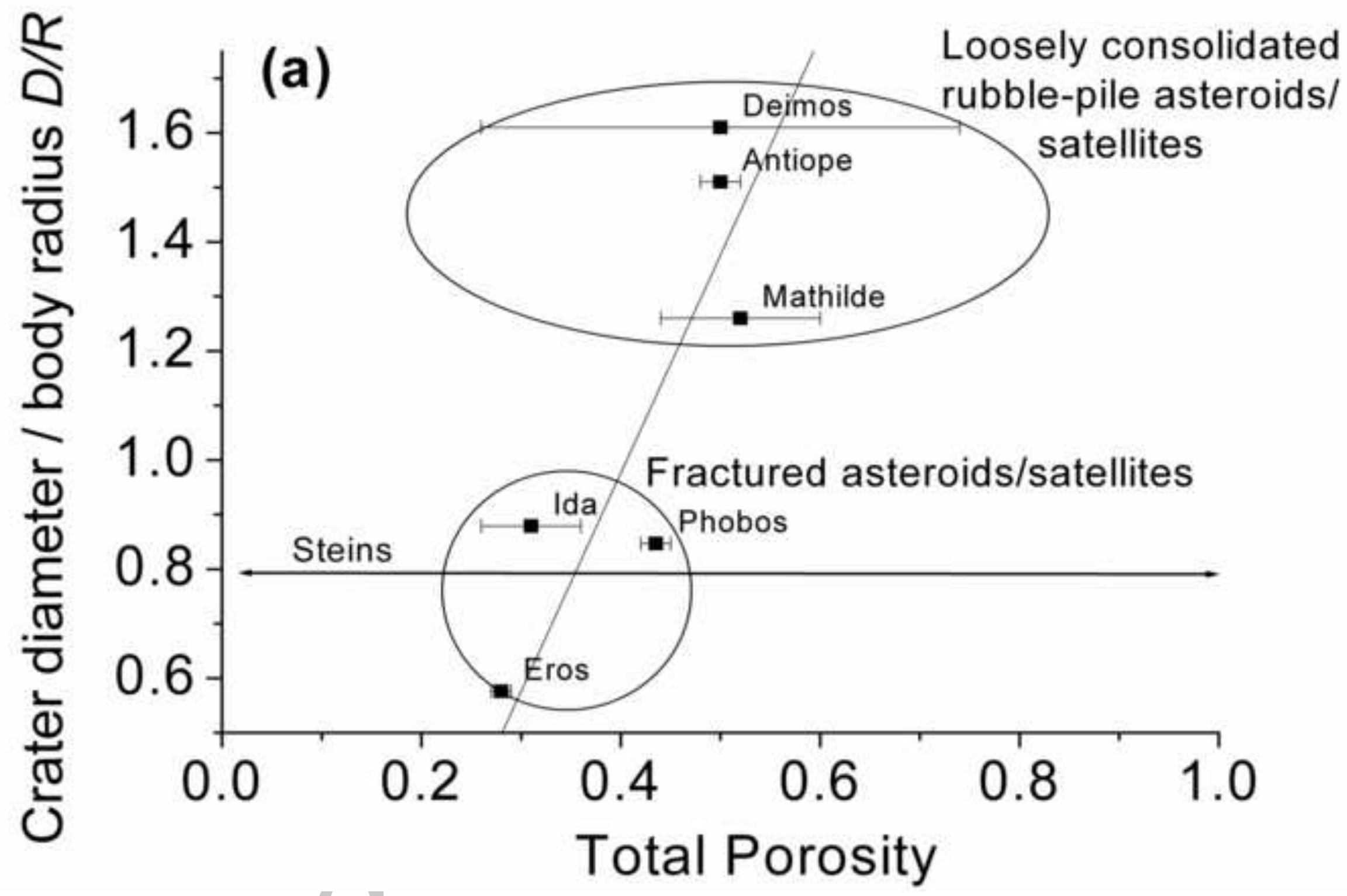




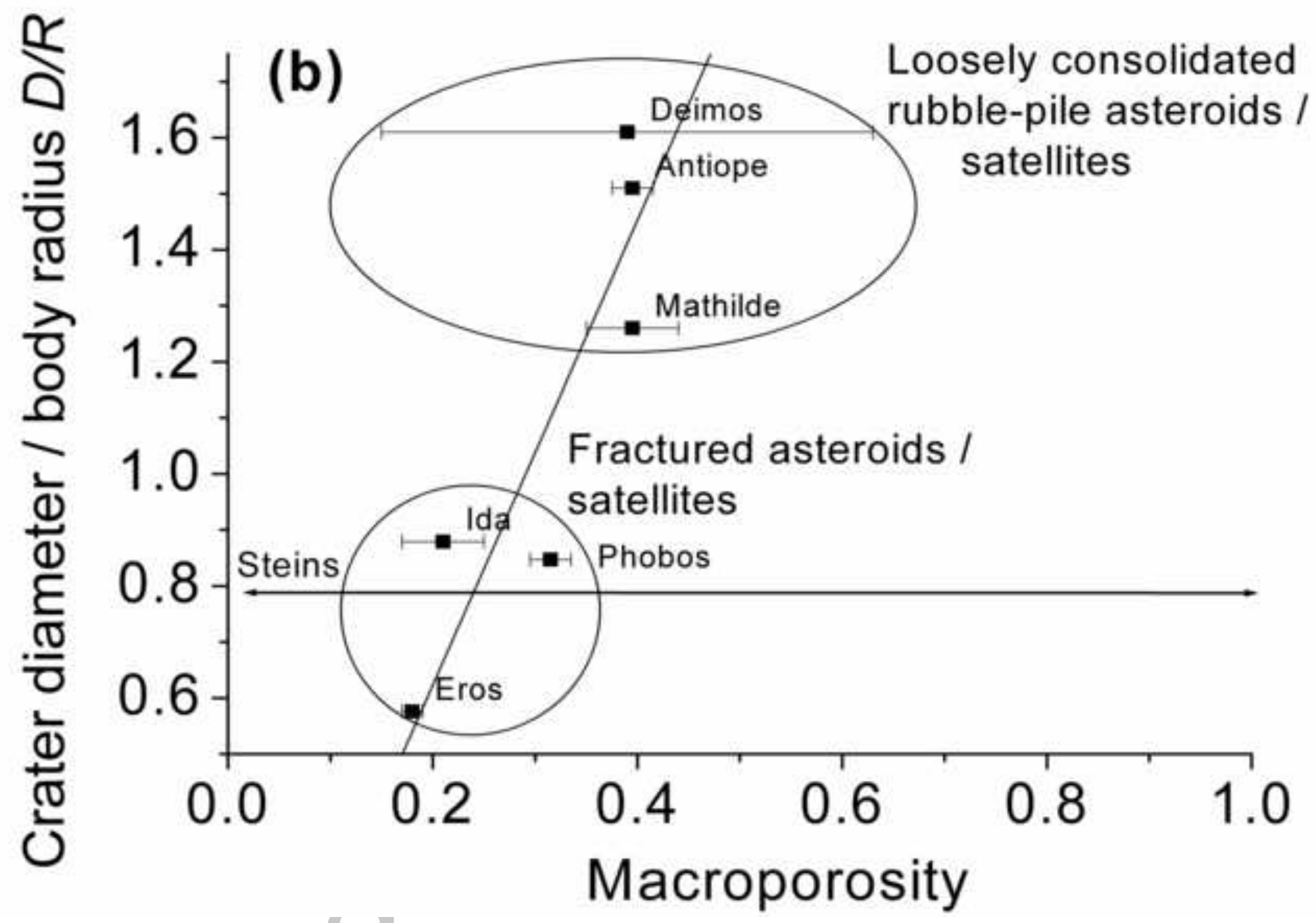

